\title{
ПРИКЛАДНАЯ МАТЕМАТИКА
}

\author{
УДК 519.853 \\ MSC 49J52
}

\section{Исчисление коэкзостеров второго порядка*}

\section{M. Э. Аббасов}

Санкт-Петербургский государственный университет, Российская Федерация, 1199034, Санкт-Петербург, Университетская наб., 7-9

Для цитирования: Аббасов М. Э. Исчисление коэкзостеров второго порядка // Вестник Санкт-Петербургского университета. Прикладная математика. Информатика. Процессы управления. 2018. Т. 14. Вып. 4. С. 276-285. https://doi.org/10.21638/11702/spbu10.2018.401

Коэкзостеры - новое понятие в негладком анализе, позволяющее исследовать экстремальные свойства широкого класса функций. Этот класс вводится конструктивным образом. Аналогично «классическому» гладкому случаю здесь разработаны формулы исчисления. Коэкзостеры - семейства выпуклых компактов, дающие возможность аппроксимировать приращение изучаемой функции в окрестности рассматриваемой точки в виде либо максимина, либо минимакса аффинных функций. Для более тонкого исследования негладких функций было введено понятие коэкзостеров второго порядка, которые также являются семействами выпуклых компактов и применяются для представления аппроксимации приращения функции в виде максимина или минимакса квадратичных функций. Эти объекты используются для построения оптимизационных алгоритмов второго порядка. Однако вновь возникает важная для практики задача построения исчисления, решению которой и посвящена данная работа.

Ключевые слова: негладкий анализ, недифференцируемая оптимизация, коэкзостеры второго порядка.

Введение. Коэкзостеры - новое понятие в негладком анализе, позволяющее исследовать экстремальные свойства широкого класса непрерывных функций.

Рассмотрим функцию $f: X \rightarrow \mathbb{R}, X \subset \mathbb{R}^{n}$ - открытое множество. Пусть $x \in X$ и функция $f$ непрерывна в точке $x$.

Говорят, что в точке $x$ у функции $f$ существует верхний коэкзостер первого порядка в смысле Дини, если имеет место разложение

* Работа выполнена при финансовой поддержке Российского фонда фундаментальных исследований (проект № 18-31-00014).

(C) Санкт-Петербургский государственный университет, 2018 


$$
f(x+\Delta)=f(x)+\min _{C \in \bar{E}(x)} \max _{[a, v] \in C}[a+(v, \Delta)]+\omega_{1}(x, \Delta),
$$

в котором $\bar{E}(x)$ - семейство выпуклых компактов в $\mathbb{R}^{n+1}$, а $\omega_{1}(x, \Delta)$ удовлетворяет условию

$$
\lim _{\alpha \downarrow 0} \frac{\omega_{1}(x, \Delta)}{\alpha}=0 \quad \forall \Delta \in \mathbb{R}^{n} .
$$

Если $\omega_{1}(x, \Delta)$ в $(1)$ удовлетворяет условию

$$
\lim _{\|\Delta\| \rightarrow 0} \frac{\omega_{1}(x, \Delta)}{\|\Delta\|}=0
$$

то говорят, что в точке $x$ у функции $f$ есть верхний коэкзостер первого порядка в смысле Адамара. Множество $\bar{E}(x)$ называется верхним коэкзостером функции $f$ в точке $x$.

Говорят, что в точке $x$ у функции $f$ есть нижний коэкзостер первого порядка в смысле Дини (Адамара), если имеет место разложение

$$
f(x+\Delta)=f(x)+\max _{C \in \underline{E}(x)} \min _{[b, w] \in C}[b+(v, \Delta)]+\omega_{1}(x, \Delta),
$$

в котором $\underline{E}(x)$ - семейство выпуклых компактов в $\mathbb{R}^{n+1}$, а $\omega_{1}(x, \Delta)$ удовлетворяет $(2)$ (или $(3))$. Множество $\underline{E}(x)$ называется нижним коэкзостером первого порядка функции $f$ в точке $x$.

Понятие коэкзостеров первого порядка было введено в $[1,2]$. Условия экстремума в терминах этих объектов были получены в $[3,4]$. Исчисление для данных семейств описано в [5]. Можно выделить класс функций, имеющих непрерывное коэкзостерное отображение, что важно для оптимизационных алгоритмов, использующих эти объекты.

Для более тонкого исследования негладких функций было введено понятие коэкзостеров второго порядка $[3,6]$.

Говорят, что в точке $x$ функция $f$ обладает верхним коэкзостером второго порядка в смысле Дини, если справедливо представление

$$
f(x+\Delta)=f(x)+\min _{C \in \bar{E}(x)} \max _{[a, v, A] \in C}\left[a+\langle v, \Delta\rangle+\frac{1}{2}\langle A \Delta, \Delta\rangle\right]+\omega_{2}(x, \Delta),
$$

где

$$
\lim _{\alpha \downarrow 0} \frac{\omega_{2}(x, \alpha \Delta)}{\alpha^{2}}=0 \quad \forall \Delta \in \mathbb{R}^{n},
$$

$\bar{E}(x)$ - семейство выпуклых компактов в $\mathbb{R} \times \mathbb{R}^{n} \times \mathbb{R}^{n \times n}$. Если в (4) условие

$$
\lim _{\|\Delta\| \rightarrow 0} \frac{\omega_{2}(x, \Delta)}{\|\Delta\|^{2}}=0
$$

то говорят, что в точке $x$ функция $f$ имеет верхний коэкзостер второго порядка в смысле Адамара. Множество $\bar{E}(x)$ называется верхним коэкзостером второго порядка (в смысле Дини или Адамара) функции $f$ в точке $x$.

Аналогично говорят, что в точке $x$ у функции $f$ есть нижний коэкзостер в смысле Дини второго порядка, если справедливо представление

$$
f(x+\Delta)=f(x)+\max _{C \in \underline{E}(x)} \min _{[a, v, A] \in C}\left[a+\langle v, \Delta\rangle+\frac{1}{2}\langle A \Delta, \Delta\rangle\right]+\omega_{2}(x, \Delta),
$$


в котором $\omega_{2}(x, \Delta)$ удовлетворяет (5) для всех $\Delta \in \mathbb{R}^{n}, \underline{E}(x)$ - семейство выпуклых компактов в $\mathbb{R} \times \mathbb{R}^{n} \times \mathbb{R}^{n \times n}$. Если в $(7) \omega_{2}(x, \Delta)$ удовлетворяет (6), то в точке $x$ функция $f$ имеет нижний коэкзостер в смысле Адамара второго порядка. Множество $\underline{E}(x)$ называется нижним коэкзостером второго порядка (в смысле Дини или Адамара) функции $f$ в точке $x$.

Если существуют верхний и нижний коэкзостеры второго порядка в смысле Дини (Адамара), то пару множеств $E(x)=[\underline{E}(x) ; \bar{E}(x)]$ называют бикоэкзостером второго порядка в смысле Дини (Адамара).

Получим основные формулы исчисления коэкзостеров второго порядка.

\section{Исчисление коэкзостеров второго порядка.}

Теорема 1. Пусть у функиий $f_{1}, f_{2}: X \rightarrow \mathbb{R}$, где $X \subset \mathbb{R}^{n}-$ открытое множество, в точке $x \in X$ есть бикоэкзостеры второго порядка в смысле Дини (Адамаpa) $E_{1}(x)=\left[\underline{E}_{1}(x), \bar{E}_{1}(x)\right], E_{2}(x)=\left[\underline{E}_{2}(x), \bar{E}_{2}(x)\right]$ соответственно. Тогда фучнкия $F=f_{1}+f_{2}$ в точке $x$ также имеет бикоэкзостер второго порядка в смысле Дини (Адамара) вида $E(x)=[\underline{E}(x), \bar{E}(x)]$, где

$$
E(x)=E_{1}(x)+E_{2}(x)=\left[\underline{E}_{1}(x)+\underline{E}_{2}(x), \bar{E}_{1}(x)+\bar{E}_{2}(x)\right] .
$$

Теорема 2. Пусть функиия $f_{1}: X \rightarrow \mathbb{R}$, где $X \subset \mathbb{R}^{n}$ - открытое множество, в точке $x \in X$ имеет бикоэкзостер второго порядка в смысле Дини (Адамара) $E_{1}(x)=\left[\underline{E}_{1}(x), \bar{E}_{1}(x)\right]$, а $\lambda-$ произвольное вещественное число. Тогда у функции $F=\lambda f_{1}$ в точке $x$ также есть бикоэкзостер второго порядка в смысле Дини (Адамара) вида

$$
E(x)=[\underline{E}(x), \bar{E}(x)]= \begin{cases}{\left[\lambda \underline{E}_{1}(x), \lambda \bar{E}_{1}(x)\right],} & \text { если } \lambda \geqslant 0 \\ {\left[\lambda \bar{E}_{1}(x), \lambda \underline{E}_{1}(x)\right],} & \text { если } \lambda \leqslant 0 .\end{cases}
$$

На основании теорем 1 и 2 можно заключить, что имеет место следующее утверждение.

Теорема 3. Пусть у функиий $f_{1}, f_{2}: X \rightarrow \mathbb{R}$, где $X \subset \mathbb{R}^{n}$ - открытое множество, в точке $x \in X$ есть бикоэкзостеры второго порядка в смысле Дини (Адамара)

$$
E_{1}(x)=\left[\underline{E}_{1}(x), \bar{E}_{1}(x)\right], E_{2}(x)=\left[\underline{E}_{2}(x), \bar{E}_{2}(x)\right]
$$

соответственно, а $\lambda_{1}, \lambda_{2}$ - произвольные вещественные числа. Тогда у функции $F=$ $\lambda_{1} f_{1}+\lambda_{2} f_{2}$ в точке $x$ есть бикоэкзостер второго порядка в смысле Дини (Адамара) вида $E(x)=[\underline{E}(x), \bar{E}(x)]$, где $E(x)=\lambda_{1} E_{1}(x)+\lambda_{2} E_{2}(x)$.

Говорят, что семейства $\underline{E}_{i}(x), \bar{E}_{i}(x), i=1,2$, ограничены в совокупности константой $R>0$, если при любом $\bar{C}$ из $\underline{E}_{i}(x)$ или $\bar{E}_{i}(x), i=1,2$, выполняется неравенство

$$
|a|+\|v\|+\|A\|<R \quad \forall[a, v, A] \in C .
$$

Отметим, что здесь используются евклидова норма и норма Фробениуса. Очевидно, отсюда следует, что для каждого $i=1,2$ справедливо неравенство

$$
\left|a+\langle v, \Delta\rangle+\frac{1}{2}\langle\Delta, A \Delta\rangle\right|<R \quad \forall \Delta \in B_{1}\left(\mathbf{0}_{n}\right), \quad \forall[a, v, A] \in C,
$$

для любого $C$ из $\underline{E}_{i}(x)$ или $\bar{E}_{i}(x), i=1,2$. Здесь $B_{1}\left(\mathbf{0}_{n}\right)$ - замкнутый шар из $\mathbb{R}^{n}$ единичного радиуса с центром в начале координат. 
Теорема 4. Пусть у функиий $f_{1}, f_{2}: X \rightarrow \mathbb{R}$, где $X \subset \mathbb{R}^{n}$ - открытое множество, в точке $x \in X$ есть бикоэкзостеры второго порядка в смысле Дини (Адамара) $E_{1}(x)=\left[\underline{E}_{1}(x), \bar{E}_{1}(x)\right], E_{2}(x)=\left[\underline{E}_{2}(x), \bar{E}_{2}(x)\right]$ соответственно, причем семейства $\underline{E}_{i}(x), \bar{E}_{i}(x), i=1,2$, ограничены в совокупности константой $R$. Тогда у функции $F=f_{1} \cdot f_{2}$ в точке $x$ также есть бикоэкзостер второго порядка в смысле Дини (Адамара) вида $E(x)=[\underline{E}(x), \bar{E}(x)], u$

$$
\begin{aligned}
& \bar{E}(x)=-R\left(\underline{E}_{1}(x)+\underline{E}_{2}(x)\right)+\widetilde{\widetilde{E}}(x)+f_{1} \bar{E}_{2}(x)+f_{2} \bar{E}_{1}(x), \\
& \underline{E}(x)=-R\left(\bar{E}_{1}(x)+\bar{E}_{2}(x)\right)+\underline{\widetilde{E}}(x)+f_{1} \underline{E}_{2}(x)+f_{2} \underline{E}_{1}(x),
\end{aligned}
$$

где

$$
\begin{gathered}
\widetilde{\widetilde{E}}(x)=\left\{C=[a, v, A] \mid a=a_{1} a_{2}+R\left(a_{1}+a_{2}\right),\right. \\
\left.v=\left(v_{1}+v_{2}\right) R+a_{1} v_{2}+a_{2} v_{1}, A=A_{1}\left(a_{2}+R\right)+A_{2}\left(a_{1}+R\right)+2 v_{1} v_{2}^{T}\right\}, \\
{\left[a_{1}, v_{1}, A_{1}\right] \in C_{1} \in \bar{E}_{1}(x),\left[a_{2}, v_{2}, A_{2}\right] \in C_{2} \in \bar{E}_{2}(x),} \\
\underline{\widetilde{E}}(x)=\left\{C=[a, v, A] \mid a=a_{1} a_{2}+R\left(a_{1}+a_{2}\right),\right. \\
\left.v=\left(v_{1}+v_{2}\right) R+a_{1} v_{2}+a_{2} v_{1}, A=A_{1}\left(a_{2}+R\right)+A_{2}\left(a_{1}+R\right)+2 v_{1} v_{2}^{T}\right\}, \\
{\left[a_{1}, v_{1}, A_{1}\right] \in C_{1} \in \underline{E}_{1}(x), \quad\left[a_{2}, v_{2}, A_{2}\right] \in C_{2} \in \underline{E}_{2}(x) .}
\end{gathered}
$$

Д о к а з а т е л ь с т в о. Коэкзостеры описывают локальную аппроксимацию исследуемой функции в окрестности рассматриваемой точки, поэтому без ограничения общности можно считать, что $\Delta \in B_{\delta}\left(\mathbf{0}_{n}\right)$, где $\delta \in(0,1)$ - некоторое малое число, а $B_{\delta}\left(\mathbf{0}_{n}\right)$ - шар радиуса $\delta$ с центром в начале координат.

Имеем равенство

$$
\begin{aligned}
& \quad F(x+\Delta)=f_{1}(x+\Delta) \cdot f_{2}(x+\Delta)=f_{1}(x) \cdot f_{2}(x)+ \\
& +\min _{C \in \bar{E}_{1}(x)} \max _{[a, v, A] \in C}\left[a+\langle v, \Delta\rangle+\frac{1}{2}\langle A \Delta, \Delta\rangle\right] \times \\
& \times \min _{C \in \bar{E}_{2}(x)} \max _{[a, v, A] \epsilon C}\left[a+\langle v, \Delta\rangle+\frac{1}{2}\langle A \Delta, \Delta\rangle\right]+ \\
& +f_{1}(x) \cdot \min _{C \in \bar{E}_{2}(x)} \min _{a, v, A] \in C}\left[a+\langle v, \Delta\rangle+\frac{1}{2}\langle A \Delta, \Delta\rangle\right]+ \\
& +f_{2}(x) \cdot \min _{C \in \bar{E}_{1}(x)} \max _{[a, v, A] \in C}\left[a+\langle v, \Delta\rangle+\frac{1}{2}\langle A \Delta, \Delta\rangle\right]+\omega_{2}(x, \Delta),
\end{aligned}
$$

где $\omega_{2}(x, \Delta)$ удовлетворяет (5) или $(6)$ в зависимости от того, в каком смысле заданы бикоэкзостеры второго порядка $E_{1}(x), E_{2}(x)$.

Очевидно, для любого $\delta \in(0,1)$ и для каждого $i=1,2$ справедливо неравенство

$$
\left|a+\langle v, \Delta\rangle+\frac{1}{2}\langle\Delta, A \Delta\rangle\right|<R, \quad \forall \Delta \in B_{\delta}\left(\mathbf{0}_{n}\right), \quad \forall[a, v, A] \in C, \forall C \in \bar{E}_{i}(x) .
$$

Для первого слагаемого в (10) имеем выражение 


$$
\begin{aligned}
& \min _{C \in \bar{E}_{1}(x)} \max _{[a, v, A] \in C}\left[a+\langle v, \Delta\rangle+\frac{1}{2}\langle\Delta, A \Delta\rangle\right] \times \\
& \times \min _{C \in \bar{E}_{2}(x)} \max _{[a, v, A] \in C}\left[a+\langle v, \Delta\rangle+\frac{1}{2}\langle\Delta, A \Delta\rangle\right]= \\
& =\left(\min _{C \in \bar{E}_{1}(x)} \max _{[a, v, A] \in C}\left[a+\langle v, \Delta\rangle+\frac{1}{2}\langle\Delta, A \Delta\rangle+R\right]-R\right) \times \\
& \times\left(\min _{C \in \bar{E}_{2}(x)} \max _{[a, v, A] \in C}\left[a+\langle v, \Delta\rangle+\frac{1}{2}\langle\Delta, A \Delta\rangle+R\right]-R\right)= \\
& =\min _{C_{1} \in \bar{E}_{1}(x), C_{2} \in \bar{E}_{2}(x)\left[a_{1}, v_{1}, A_{1}\right] \in C_{1},\left[a_{2}, v_{2}, A_{2}\right] \in C_{2}}\left[\left(a_{1}+\left\langle v_{1}, \Delta\right\rangle+\right.\right. \\
& \left.+\frac{1}{2}\left\langle\Delta, A_{1} \Delta\right\rangle\right)\left(a_{2}+\left\langle v_{2}, \Delta\right\rangle+\frac{1}{2}\left\langle\Delta, A_{2} \Delta\right\rangle\right)+ \\
& \left.+R\left(a_{1}+a_{2}+\left\langle v_{1}+v_{2}, \Delta\right\rangle+\frac{1}{2}\left\langle\Delta,\left(A_{1}+A_{2}\right) \Delta\right\rangle\right)\right]- \\
& \text { - } R \min _{C_{1} \in \bar{E}_{1}(x), C_{2} \in \bar{E}_{2}(x)\left[a_{1}, v_{1}, A_{1}\right] \in C_{1},\left[a_{2}, v_{2}, A_{2}\right] \in C_{2}}\left(a_{1}+a_{2}+\right. \\
& \left.+\left\langle v_{1}+v_{2}, \Delta\right\rangle+\frac{1}{2}\left\langle\Delta,\left(A_{1}+A_{2}\right) \Delta\right\rangle\right) .
\end{aligned}
$$

Для первого слагаемого в (11) можно записать, что

$$
\begin{gathered}
\min _{C_{1} \in \bar{E}_{1}(x), C_{2} \in \bar{E}_{2}(x)\left[a_{1}, v_{1}, A_{1}\right] \in C_{1},\left[a_{2}, v_{2}, A_{2}\right] \in C_{2}}\left[\left(a_{1}+\left\langle v_{1}, \Delta\right\rangle+\right.\right. \\
\left.+\frac{1}{2}\left\langle\Delta, A_{1} \Delta\right\rangle\right)\left(a_{2}+\left\langle v_{2}, \Delta\right\rangle+\frac{1}{2}\left\langle\Delta, A_{2} \Delta\right\rangle\right)+ \\
\left.+R\left(a_{1}+a_{2}+\left\langle v_{1}+v_{2}, \Delta\right\rangle+\frac{1}{2}\left\langle\Delta,\left(A_{1}+A_{2}\right) \Delta\right\rangle\right)\right]= \\
=\min _{C_{1} \in \bar{E}_{1}(x), C_{2} \in \bar{E}_{2}(x)\left[a_{1}, v_{1}, A_{1}\right] \in C_{1},\left[a_{2}, v_{2}, A_{2}\right] \in C_{2}}\left[a_{1} a_{2}+R\left(a_{1}+a_{2}\right)+\right. \\
+\left\langle a_{1} v_{2}+a_{2} v_{1}+R\left(v_{1}+v_{2}\right), \Delta\right\rangle+ \\
\left.+\frac{1}{2}\left\langle\Delta,\left(a_{1} A_{2}+a_{2} A_{1}+2 v_{1} v_{2}^{T}+R\left(A_{1}+A_{2}\right)\right) \Delta\right\rangle\right]+\omega_{2}(x, \Delta)= \\
=\min _{C \in \widetilde{E}(x)} \max _{a, v, A] \epsilon C}\left[a+\langle v, \Delta\rangle+\frac{1}{2}\langle\Delta, A \Delta\rangle\right]+\omega_{2}(x, \Delta),
\end{gathered}
$$

здесь

$$
\begin{gathered}
\widetilde{\bar{E}}(x)=\left\{C=[a, v, A] \mid a=a_{1} a_{2}+R\left(a_{1}+a_{2}\right),\right. \\
\left.v=\left(v_{1}+v_{2}\right) R+a_{1} v_{2}+a_{2} v_{1}, A=A_{1}\left(a_{2}+R\right)+A_{2}\left(a_{1}+R\right)+2 v_{1} v_{2}^{T}\right\}, \\
{\left[a_{1}, v_{1}, A_{1}\right] \in C_{1} \in \bar{E}_{1}(x),\left[a_{2}, v_{2}, A_{2}\right] \in C_{2} \in \bar{E}_{2}(x),}
\end{gathered}
$$




$$
\begin{aligned}
& \min _{C_{1} \in \bar{E}_{1}(x), C_{2} \in \bar{E}_{2}(x)\left[a_{1}, v_{1}, A_{1}\right] \in C_{1},\left[a_{2}, v_{2}, A_{2}\right] \in C_{2}} \psi\left(x, \Delta, v_{1}, A_{1}, v_{2}, A_{2}\right) \leqslant \\
& \leqslant \min _{2}(x, \Delta) \leqslant \\
& \leqslant \max _{C_{1} \in \bar{E}_{1}(x), C_{2} \in \bar{E}_{2}(x)\left[a_{1}, v_{1}, A_{1}\right] \in C_{1},\left[a_{2}, v_{2}, A_{2}\right] \in C_{2}} \psi\left(x, \Delta, v_{1}, A_{1}, v_{2}, A_{2}\right),
\end{aligned}
$$

$$
\psi\left(x, \Delta, v_{1}, A_{1}, v_{2}, A_{2}\right)=\frac{1}{2}\left\langle v_{1}, \Delta\right\rangle\left\langle\Delta, A_{2} \Delta\right\rangle+\frac{1}{2}\left\langle v_{2}, \Delta\right\rangle\left\langle\Delta, A_{1} \Delta\right\rangle+\frac{1}{4}\left\langle\Delta, A_{1} \Delta\right\rangle\left\langle\Delta, A_{2} \Delta\right\rangle .
$$

Отметим, что, в силу ограниченности в совокупности семейств $\underline{E}_{i}(x), \bar{E}_{i}(x), i=$ 1,2 , стремление

$$
\frac{\psi\left(x, \Delta, v_{1}, A_{1}, v_{2}, A_{2}\right)}{\|\Delta\|^{2}} \underset{\|\Delta\| \rightarrow 0}{\longrightarrow} 0
$$

будет равномерным по совокупности переменных $v_{1}, A_{1}, v_{2}, A_{2}$, где $\left[a_{1}, v_{1}, A_{1}\right] \in C_{1} \epsilon$ $\bar{E}_{1}(x),\left[a_{2}, v_{2}, A_{2}\right] \in C_{2} \in \bar{E}_{2}(x)$, а потому $\omega_{2}(x, \Delta)$ в (12) удовлетворяет условию $(6)$.

Для $i=1,2$ имеем (см. (4), (7)) выражение

$$
\begin{aligned}
& \min _{C \in \bar{E}_{i}(x)} \max _{[a, v, A] \in C}\left[a+\langle v, \Delta\rangle+\frac{1}{2}\langle\Delta, A \Delta\rangle\right]= \\
& \quad=\max _{C \in \underline{E}_{i}(x)} \min _{[a, v, A] \in C}\left[a+\langle v, \Delta\rangle+\frac{1}{2}\langle\Delta, A \Delta\rangle\right]+\omega_{2}(x, \Delta) .
\end{aligned}
$$

Складывая равенства (13) при $i=1$ и $i=2$, получаем

$$
\begin{gathered}
\min _{C_{1} \in \bar{E}_{1}(x), C_{2} \in \bar{E}_{2}(x)\left[a_{1}, v_{1}, A_{1}\right] \in C_{1},\left[a_{2}, v_{2}, A_{2}\right] \in C_{2}}\left(a_{1}+a_{2}+\right. \\
\left.+\left\langle v_{1}+v_{2}, \Delta\right\rangle+\frac{1}{2}\left\langle\Delta,\left(A_{1}+A_{2}\right) \Delta\right\rangle\right)= \\
=\min _{C_{1} \in \underline{E}_{1}(x), C_{2} \in \underline{E}_{2}(x)\left[a_{1}, v_{1}, A_{1}\right] \in C_{1},\left[a_{2}, v_{2}, A_{2}\right] \in C_{2}}\left(a_{1}+a_{2}+\left\langle v_{1}+v_{2}, \Delta\right\rangle+\right. \\
\left.+\frac{1}{2}\left\langle\Delta,\left(A_{1}+A_{2}\right) \Delta\right\rangle\right)+\omega_{2}(x, \Delta) .
\end{gathered}
$$

Для второго слагаемого в (11) с учетом (14) имеем цепочку равенств

$$
\begin{gathered}
-R \min _{C_{1} \in \bar{E}_{1}(x), C_{2} \in \bar{E}_{2}(x)\left[a_{1}, v_{1}, A_{1}\right] \in C_{1},\left[a_{2}, v_{2}, A_{2}\right] \in C_{2}}\left(a_{1}+a_{2}+\right. \\
\left.+\left\langle v_{1}+v_{2}, \Delta\right\rangle+\frac{1}{2}\left\langle\Delta,\left(A_{1}+A_{2}\right) \Delta\right\rangle\right)= \\
=-R \min _{C_{1} \in \underline{E}_{1}(x), C_{2} \in \underline{E}_{2}(x)\left[a_{1}, v_{1}, A_{1}\right] \in C_{1},\left[a_{2}, v_{2}, A_{2}\right] \in C_{2}}\left(a_{1}+a_{2}+\right. \\
\left.+\left\langle v_{1}+v_{2}, \Delta\right\rangle+\frac{1}{2}\left\langle\Delta,\left(A_{1}+A_{2}\right) \Delta\right\rangle\right)+\omega_{2}(x, \Delta)=
\end{gathered}
$$




$$
\begin{aligned}
& =-R \max _{C \in \underline{E}_{1}(x)+\underline{E}_{2}(x)[a, v, A] \in C} \min _{=}\left[a+\langle v, \Delta\rangle+\frac{1}{2}\langle\Delta, A \Delta\rangle\right]+\omega_{2}(x, \Delta)= \\
& =\min _{C \in \underline{E}_{1}(x)+\underline{E}_{2}(x)}\left[-R \min _{[a, v, A] \in C}\left[a+\langle v, \Delta\rangle+\frac{1}{2}\langle\Delta, A \Delta\rangle\right]+\omega_{2}(x, \Delta)=\right. \\
& =\min _{C \in \underline{E}_{1}(x)+\underline{E}_{2}(x)[a, v, A] \in C}\left[-R\left(a+\langle v, \Delta\rangle+\frac{1}{2}\langle\Delta, A \Delta\rangle\right]+\omega_{2}(x, \Delta)=\right. \\
& =\min _{C \in-R \underline{E}_{1}(x)-R \underline{E}_{2}(x)[a, v, A] \in C}\left[a+\langle v, \Delta\rangle+\frac{1}{2}\langle\Delta, A \Delta\rangle\right]+\omega_{2}(x, \Delta) .
\end{aligned}
$$

Итак, окончательно из (10) с учетом (11), (12), (15) получаем разложение

$$
\begin{aligned}
& F(x+\Delta)=F(x)+\min _{C \in \overline{\bar{E}}(x)} \max _{a, v, A] \epsilon C}\left[a+\langle v, \Delta\rangle+\frac{1}{2}\langle\Delta, A \Delta\rangle\right]+ \\
& +\min _{C \in-R\left(\underline{E}_{1}(x)+\underline{E}_{2}(x)\right)[a, v, A] \in C} \max _{[}\left[a+\langle v, \Delta\rangle+\frac{1}{2}\langle\Delta, A \Delta\rangle\right]+ \\
& \quad+f_{1}(x) \cdot \min _{C \in \bar{E}_{2}(x)[a, v, A] \in C} \min _{\left[a+\langle v, \Delta\rangle+\frac{1}{2}\langle A \Delta, \Delta\rangle\right]+}\left[a+f_{2}(x) \cdot \min _{C \in \bar{E}_{1}(x)} \max _{[a, v, A] \in C}\left[a+\langle v, \Delta\rangle+\frac{1}{2}\langle A \Delta, \Delta\rangle\right]+\omega_{2}(x, \Delta),\right.
\end{aligned}
$$

откуда и следует формула (8). Выражение (9) доказывается аналогично.

Теорема 5. Пусть функиия $f_{1}: X \rightarrow \mathbb{R}$, где $X \subset \mathbb{R}^{n}$ - открытое множество, в точке $x \in X$ имеет бикоэкзостер второго порлдка в смысле Дини (Адамара) $E_{1}(x)=\left[\underline{E}_{1}(x), \bar{E}_{1}(x)\right]$, причем семейства $\underline{E}_{1}(x)$ и $\bar{E}_{1}(x)$ ограничены в совокупности константой $R$ u, кроме того, $f_{1}(x) \neq 0$. Тогда и функиия $F=\frac{1}{f_{1}}$ в точке $x$ также имеет бикоэкзостер второго порядка вида в смысле Дини (Адамара) $E(x)=[\underline{E}(x), \bar{E}(x)]$, где

$$
\begin{gathered}
\underline{E}(x)=-\frac{\bar{E}_{1}(x)}{f_{1}^{2}(x)}+\frac{1}{f_{1}^{3}(x)}\left(-2 R \bar{E}(x)+2 f_{1}(x) \underline{E}_{1}(x)+\underline{\widetilde{E}}(x)\right), \\
\bar{E}(x)=-\frac{\underline{E}_{1}(x)}{f_{1}^{2}(x)}+\frac{1}{f_{1}^{3}(x)}\left(-2 R \underline{E}(x)+2 f_{1}(x) \bar{E}_{1}(x)+\widetilde{\bar{E}}(x)\right), \\
\widetilde{\bar{E}}(x)=\left\{C=[a, v, A] \mid a=a_{1} a_{2}+R\left(a_{1}+a_{2}\right),\right. \\
\left.v=\left(v_{1}+v_{2}\right) R+a_{1} v_{2}+a_{2} v_{1}, A=A_{1}\left(a_{2}+R\right)+A_{2}\left(a_{1}+R\right)+2 v_{1} v_{2}^{T}\right\}, \\
{\left[a_{1}, v_{1}, A_{1}\right] \in C_{1} \in \bar{E}_{1}(x),\left[a_{2}, v_{2}, A_{2}\right] \in C_{2} \in \bar{E}_{1}(x),} \\
\widetilde{E}(x)=\left\{C=[a, v, A] \mid a=a_{1} a_{2}+R\left(a_{1}+a_{2}\right),\right. \\
\left.v=\left(v_{1}+v_{2}\right) R+a_{1} v_{2}+a_{2} v_{1} . A=A_{1}\left(a_{2}+R\right)+A_{2}\left(a_{1}+R\right)+2 v_{1} v_{2}^{T}\right\}, \\
{\left[a_{1}, v_{1}, A_{1}\right] \in C_{1} \in \underline{E}_{1}(x),\left[a_{2}, v_{2}, A_{2}\right] \in C_{2} \in \underline{E}_{1}(x) .}
\end{gathered}
$$

До к а з а т е л ь с т в о. Поскольку

$$
\frac{1}{z+\alpha}=\frac{1}{z}-\frac{1}{z^{2}} \alpha+\frac{1}{z^{3}} \alpha^{2}+o\left(\alpha^{2}\right)
$$

то с учетом теоремы 4 получаем требуемое. 
Теорема 6. Пусть $I-$ конечное множество индексов, $X \subset \mathbb{R}^{n}-$ открытое множество, функиии $f_{i}(x): X \rightarrow \mathbb{R}, i \in I$, имеют бикоэкзостеры второго порядка в смысле Дини (Адамара) $E_{i}\left(x_{0}\right)=\left[\underline{E}_{i}\left(x_{0}\right), \bar{E}_{i}\left(x_{0}\right)\right]$. Тогда

1) у функиии $F_{1}(x)=\max _{i \in I} f_{i}(x)$ в точке $x_{0}$ есть нижний коэкзостер второго порядка в смысле Дини (Адамара)

$$
\underline{E}_{1}\left(x_{0}\right)=\bigcup_{i \in I} \widehat{E}_{i}\left(x_{0}\right),
$$

əде

$$
\underline{\underline{E}}_{i}\left(x_{0}\right)=\left\{C+\left[f_{i}\left(x_{0}\right)-F_{1}\left(x_{0}\right), \mathbf{0}_{n}, \mathbf{0}_{n \times n}\right] \mid C \in \underline{E}_{i}\left(x_{0}\right)\right\} ;
$$

2) у функции $F_{2}(x)=\min _{i \in I} f_{i}(x)$ в точке $x_{0}$ есть верхний коэкзостер второго порядка в смысле Дини (Адамара)

$$
\bar{E}_{2}\left(x_{0}\right)=\bigcup_{i \in I} \widehat{\bar{E}}_{y}\left(x_{0}\right),
$$

здесъ

$$
\widehat{\bar{E}}_{y}\left(x_{0}\right)=\left\{C+\left[f_{i}\left(x_{0}\right)-F_{2}\left(x_{0}\right), \mathbf{0}_{n}, \mathbf{0}_{n \times n}\right] \mid C \in \bar{E}_{i}\left(x_{0}\right)\right\}
$$

Д о к а з а т е л ь с т в о. Имеем цепочку равенств

$$
\begin{gathered}
F_{1}\left(x_{0}+\Delta\right)=\max _{i \in I} f_{i}\left(x_{0}+\Delta\right)=\max _{i \in I} f_{i}\left(x_{0}\right)+\max _{i \in I}\left[f_{i}\left(x_{0}\right)-\max _{i \in I} f_{i}\left(x_{0}\right)+\right. \\
\left.+\max _{C \in \underline{E}_{i}\left(x_{0}\right)} \min _{[a, v, A] \in C}\left[a+\langle v, \Delta\rangle+\frac{1}{2}\langle A \Delta, \Delta\rangle\right]+\omega_{2, i}\left(x_{0}, \Delta\right)\right]= \\
=F_{1}\left(x_{0}\right)+\max _{i \in I}\left[\psi_{i}\left(x_{0}, \Delta\right)+\omega_{2, i}\left(x_{0}, \Delta\right)\right],
\end{gathered}
$$

для упрощения записи используем обозначение

$$
\psi_{i}\left(x_{0}, \Delta\right)=f_{i}\left(x_{0}\right)-\max _{i \in I} f_{i}\left(x_{0}\right)+\max _{C \in \underline{E}_{i}\left(x_{0}\right)} \min _{[a, v, A] \in C}\left[a+\langle v, \Delta\rangle+\frac{1}{2}\langle A \Delta, \Delta\rangle\right] .
$$

Заметим, что, с одной стороны,

$$
\max _{i \in I}\left[\psi_{i}\left(x_{0}, \Delta\right)+\omega_{2, i}\left(x_{0}, \Delta\right)\right] \leqslant \max _{i \in I} \psi_{i}\left(x_{0}, \Delta\right)+\max _{i \in I} \omega_{2, i}\left(x_{0}, \Delta\right),
$$

а с другой -

$$
\begin{aligned}
\max _{i \in I}\left[\psi_{i}\left(x_{0}, \Delta\right)+\right. & \left.\omega_{2, i}\left(x_{0}, \Delta\right)\right] \geqslant \max _{i \in I}\left[\psi_{i}\left(x_{0}, \Delta\right)+\min _{i \in I} \omega_{2, i}\left(x_{0}, \Delta\right)\right]= \\
& =\max _{i \in I} \psi_{i}\left(x_{0}, \Delta\right)+\min _{i \in I} \omega_{2, i}\left(x_{0}, \Delta\right) .
\end{aligned}
$$

Поэтому можем записать неравенства

$$
\min _{i \in I} \omega_{2, i}\left(x_{0}, \Delta\right) \leqslant \max _{i \in I}\left[\psi_{i}\left(x_{0}, \Delta\right)+\omega_{2, i}\left(x_{0}, \Delta\right)\right]-\max _{i \in I} \psi_{i}\left(x_{0}, \Delta\right) \leqslant \max _{i \in I} \omega_{2, i}\left(x_{0}, \Delta\right),
$$

откуда получаем

$$
\max _{i \in I}\left[\psi_{i}\left(x_{0}, \Delta\right)+\omega_{2, i}\left(x_{0}, \Delta\right)\right]-\max _{i \in I} \psi_{i}\left(x_{0}, \Delta\right)=\omega_{2}\left(x_{0}, \Delta\right),
$$


где в зависимости от того, в каком смысле задавались бикоэкзостеры $E_{i}\left(x_{0}\right), i \in I$, выполняется одно из условий

$$
\lim _{\alpha \downarrow 0} \frac{\omega_{2}(x, \alpha \Delta)}{\alpha^{2}}=0 \quad \forall \Delta \in \mathbb{R}^{n}, \quad \lim _{\|\Delta\| \rightarrow 0} \frac{\omega_{2}\left(x_{0}, \Delta\right)}{\|\Delta\|^{2}}=0 .
$$

Таким образом, можно записать, что

$$
\begin{gathered}
F_{1}\left(x_{0}+\Delta\right)=F_{1}\left(x_{0}\right)+\max _{i \in I} \psi_{i}\left(x_{0}, \Delta\right)+\omega_{2}\left(x_{0}, \Delta\right)=F_{1}\left(x_{0}\right)+ \\
+\max _{C \in \bigcup_{i \in I} \underline{E}_{i}\left(x_{0}\right)} \min _{[a, v, A] \in C}\left[a+f_{i}\left(x_{0}\right)-F_{1}\left(x_{0}\right)+\langle v, \Delta\rangle+\frac{1}{2}\langle A \Delta, \Delta\rangle\right]+\omega_{2}\left(x_{0}, \Delta\right) .
\end{gathered}
$$

Для $F_{2}$ доказательство проводится таким же образом.

\section{Литература}

1. Абанъкин A. Е. Безусловная минимизация $H$-гипердифференцируемых функций // Журн. вычисл. математики и матем. физики. 1998. Т. 38(9). С. 1500-1508.

2. Demyanov V.F. Exhausters and convexificators - New tools in nonsmooth analysis // Quasidifferentiability and related topics / eds by V. Demyanov, A. Rubinov. Dordrecht: Kluwer Academic Publ., 2000. P. 85-137.

3. Abbasov M. E., Demyanov V. F. Extremum conditions for a nonsmooth function in terms of exhausters and coexhausters // Proceedings of the Steklov Institute of Mathematics. 2010. Vol. 269(1). P. 6-15.

4. Abbasov M. E., Demyanov V. F. Adjoint coexhausters in nonsmooth analysis and extremality conditions // Journal of Optimization Theory and Applications. 2013. Vol. 156(3). P. 535-553.

5. Dolgopolik M. V. Inhomogeneous convex approximations of nonsmooth functions // Russian Mathematics. 2012. Vol. 56(12). P. 28-42.

6. Abbasov M. E. Second-order minimization method for nonsmooth functions allowing convex quadratic approximations of the augment // Journal of Optimization Theory and Applications. 2016. Vol. 171(2). P. 666-674.

Статья поступила в редакцию 23 марта 2018 г.

Статья принята к печати 25 сентября 2018 г.

Контактная информация:

Аббасов Меджид Элъхан огль - канд. физ.-мат. наук, доц.; m.abbasov@spbu.ru

\section{Calculus of second order coexhausters*}

\section{E. Abbasov}

St. Petersburg State University, 7-9, Universitetskaya nab., St. Petersburg, 199034, Russian Federation

For citation: Abbasov M. E. Calculus of second order coexhausters. Vestnik of Saint Petersburg University. Applied Mathematics. Computer Science. Control Processes, 2018, vol. 14, iss. 4, pp. 276-285. https://doi.org/10.21638/11702/spbu10.2018.401 (In Russian)

Coexhasuter is a new notion in the nonsmooth analysis that allows one to study extremal properties of a wide class of functions. This class is introduced in a constructive manner analogous to the "classical" smooth case. Formulas of calculus were developed. Coexhausters are families of convex compact sets allowing one to approximate the increment of the studied function in the neighbourhood of the considered point in the form of MaxMin or MiniMax

${ }^{*}$ The work is supported by Russian Found of Fundamental Research (project N 18-31-00014 mol-a). 
of affine functions. For a more detailed study of nonsmooth functions, a notion of secondorder coexhausters was introduced. These are also families of convex compact sets which are used to represent the approximation of the increment of the studied function in the form of MaxMin or MiniMax of quadratic functions. These objects are used to build secondorder optimization algorithms. However, an important problem of constructing calculus arises again. The solution to this problem is the subject of this paper.

Keywords: nonsmooth analysis, nondifferentiable optimization, second order coexhausters.

\section{References}

1. Abankin A. E. Bezuslovnaia minimizatsiia $H$-giperdifferentsiruemykh funktsii [Unconstrained minimization of $H$-hyperdifferentiable functions]. Computational Mathematics and Mathematical Physics, 1998, vol. 38(9), pp. 1500-1508. (In Russian)

2. Demyanov V. F. Exhausters and convexificators - New tools in nonsmooth analysis. Quasi-differentiability and related topics. Eds by V. Demyanov, A. Rubinov. Dordrecht, Kluwer Academic Publ., 2000, pp. 85-137.

3. Abbasov M. E., Demyanov V. F. Extremum conditions for a nonsmooth function in terms of exhausters and coexhausters. Proceedings of the Steklov Institute of Mathematics, 2010, vol. 269(1), pp. $6-15$.

4. Abbasov M. E., Demyanov V. F. Adjoint coexhausters in nonsmooth analysis and extremality conditions. Journal of Optimization Theory and Applications, 2013, vol. 156(3), pp. 535-553.

5. Dolgopolik M. V. Inhomogeneous convex approximations of nonsmooth functions. Russian Mathematics, 2012, vol. 56(12), pp. 28-42.

6. Abbasov M. E. Second-order minimization method for nonsmooth functions allowing convex quadratic approximations of the augment. Journal of Optimization Theory and Applications, 2016, vol. 171(2), pp. 666-674.

Received: March 23, 2018.

Accepted: September 25, 2018.

Author's information:

Majid E. Abbasov - PhD in Physics and Mathematics, Associate Professor; m.abbasov@spbu.ru 